\title{
Production of synthetic tetraploids as a tool for polyploid research
}

\author{
Mariana Castro $^{1}$, Sílvia Castro ${ }^{1,2}$, and João Loureiro ${ }^{1}$ \\ ${ }^{1}$ CFE, Centre for Functional Ecology, Department of Life Sciences, University of Coimbra, \\ Calçada Martim de Freitas, 3000-456 Coimbra, Portugal \\ ${ }^{2}$ Botanic Garden of the University of Coimbra, Calçada Martim de Freitas, 3000-456 Coimbra, Portugal \\ Correspondence: Mariana Castro (mcastro@uc.pt)
}

Received: 24 April 2018 - Revised: 6 August 2018 - Accepted: 8 August 2018 - Published: 7 September 2018

\begin{abstract}
Polyploidization has been traditionally considered a phenomenon that mediates ecological differentiation; however, the adaptive value of genome duplications has been seldom explored. Natural or synthetic polyploids offer unique opportunities to experimentally quantify the immediate consequences of genome duplications in plant traits that may be involved with ecological differentiation. Jasione maritima is a diploid-tetraploid complex with cytotypes distributed allopatrically in an environmental gradient ideal for exploring the role of genome duplications in the success of polyploid lineages. However, like in numerous other polyploid complexes, neotetraploids of J. maritima have not been found in nature. In this study we developed a methodology to obtain synthetic tetraploids from seeds collected in wild diploid plants of J. maritima. We tested the effect of different colchicine concentrations and seedling ages in survival and polyploidization success of J. maritima seedlings, using flow cytometry to assess ploidy level and considering the population of origin. A methodology to synchronize seed germination was also explored. Synchronization of seed germination was best achieved using a cold treatment of 2 weeks before the transference to a growth chamber. An overall survival rate of $11.5 \pm 0.7 \%$ and further tetraploid conversion of $35.6 \pm 2.9 \%$ was obtained using 3-day-old seedlings of J. maritima. Survival rates were variable depending on colchicine treatment (the highest the most lethal) and seedling origin (i.e. population), while conversion rate was similar across these factors and high rates of tetraploid conversion were obtained. Considering that the main constraint was survival to the colchicine treatment, we suggest the use of the lowest colchicine concentration tested, i.e. $0.1 \%$ colchicine. The use of older seedlings increased survival rates but, in many cases, compromised a complete tetraploid conversion, generating many diploid-tetraploid mixoploid plants.
\end{abstract}

\section{Introduction}

Polyploidy have long been recognized to play a significant role in the evolution and diversification of flowering plants (Soltis, 2005), being correlated with explosions in species diversity (Soltis et al., 2009). Accordingly, $15 \%$ of the speciation events in angiosperms have been associated with ploidy increase (Wood et al., 2009). After polyploidization the architecture of the cell is modified, the cell must adapt to the new nuclear DNA content and deal with changes in the homology of the chromosomes, gene expression and epigenetics (Comai, 2005; Husband et al., 2013; Barker et al., 2015). Changes in gene expression and developmental processes due to genome duplications can potentially generate shifts in the morphology, reproduction and physiology of the polyploid individual (Levin, 2002). Consequently, it may provide unique or transgressive tolerances and developmental patterns, which could confer an advantage to these newly formed polyploids to conditions that are beyond the limits of their diploid (or lower ploidy) progenitors (Petit and Thompson, 1999; Manzaneda et al., 2012).

Polyploidization has been traditionally considered a phenomenon that mediates ecological differentiation; however, the adaptive value of genome duplications has been seldomly explored. Despite its importance, the majority of the stud- 
ies published so far compared pairs of congeneric species or established cytotypes of a given species, without considering the temporal scale, i.e. the time that has passed since the formation of the polyploid (e.g. Segraves and Thompson, 1999; Jersáková et al., 2010; Ramsey and Schemske, 2002; Husband and Sabara, 2004). In that sense, those studies could not determine if the observed differences (or their absence) were due to subsequent evolution of the polyploid lineage or if they were exclusively due to the duplication of the genome. The detection of newly formed polyploids in natural populations (i.e. neopolyploids) using flow cytometry (Kron et al., 2007; Ramsey, 2011), or the synthesis of polyploids in the laboratory using mutagenic anti-mitotic agents (Husband et al., 2008), enables a unique opportunity to experimentally quantify the immediate consequences of genome duplications in morphological, physiological and developmental plant traits. While the comparison between diploid (or lower ploidy) progenitors and neopolyploids (either naturally occurring or synthetized) enables us to explore the consequences of polyploidy per se, the comparison between neopolyploids and established polyploids enables us to explore the changes that accumulated after their origin (e.g. Bretagnolle and Lumaret, 1995; De Kovel and De Jong, 2000; Ramsey and Schemske, 2002; Oswald and Nuismer, 2011; Ramsey, 2011; Münzbergová, 2017; Pavlíková et al., 2017). Therefore, neopolyploids allow the immediate effects of genome duplication and its role in the establishment and persistence of the new cytotype to be evaluated, being a key component when studying the ecological processes involved in polyploid evolution.

Naturally occurring neopolyploids have been detected in a few polyploid complexes and were used to study the role of genome duplications in the successful establishment of polyploid lineages (e.g. Achillea borealis, Ramsey, 2011). However, its occurrence in nature represents a snapshot in the evolutionary history of a given polyploid group and might thus be very difficult to encounter in nature and be confined to recently formed complexes. As an alternative, researchers have developed methodologies to synthesize polyploids in the laboratory. Indeed, synthetic polyploids have long been used for plant breeding because of the advantages and new features conferred by genome duplications (Semeniuk and Arisumi, 1968; Lumaret, 1988; Levin, 2002; Tamayo-Ordóñez et al., 2016). However, ecological studies using this approach are much more recent and restricted to a few polyploid complexes of autopolyploid origin (Chamerion angustifolium, Husband et al., 2008, 2016; Maherali et al., 2009; Martin and Husband, 2012, 2013; Heuchera grossulariifolia, Oswald and Nuismer, 2011; Spartina pectinata, Kim et al., 2012; Vicia cracca, Münzbergová, 2017; Pavlíková et al., 2017).

Synthetic polyploids can be obtained by applying antimitotic agents that block the cell cycle, such as colchicine, oryzalin or trifuralin (Semeniuk and Arisumi, 1968; Lignowski and Scott, 1972; Jaskani et al., 2005; Zlesak et al., 2005; Chen et al., 2006; Allum et al., 2007). From this, colchicine is the most commonly used agent in both biotechnological and ecological studies (e.g. Chen et al., 2006; Stanys et al., 2006; Ascough et al., 2008; Husband et al., 2008; Münzbergová, 2017). Colchicine causes the depolymerization of the microtubular cytoskeleton in the early phases of metaphase, blocking the separation of chromosomes in mitoses, consequently leading to polyploidization of the cells. In higher concentrations, in a later stage, it induces polymerization of new tubulin-containing structures in c-metaphase cells, allowing the reconstitution of $4 \mathrm{C}$ nuclei and their progression into the cell cycle (Caperta et al., 2006). In crop improvement, the induction protocols are usually applied in vitro to selected elite clones subjected to different colchicine concentrations, in solid or liquid cultures (Saccharum officinarum, Heinz and Mee, 1970; Citrus, Gmitter et al., 1991; Miscanthus $\times$ giganteus, Yu et al., 2009). Contrarily, in ecological studies, synthetic polyploids are usually induced from seeds or seedlings obtained in natural populations (Husband et al., 2008; Thompsonet al., 2010; Münzbergová, 2017).

Jasione maritima (Duby) Merino (Campanulaceae) is an endemic plant from north-west dune systems of the Iberian Peninsula, closely related with J. montana, a widely distributed species in Europe (Sales and Hedge, 2001). J. maritima is a diploid-tetraploid complex with cytotypes showing an allopatric distribution, harbouring diploid populations ( $2 \mathrm{n}=2 x=12$ chromosomes) in the northern parts of the distribution range, while tetraploid populations $(2 \mathrm{n}=4 x=24)$ occur in the southern area (Castro et al., 2015; Castro, 2018). This system is ideal for addressing questions related with polyploid establishment, because the cytotypes are distributed across an environmental gradient (Castro et al., 2015; Castro, 2018) and seem to bear some differences in morphological and fitness-related traits (Lago and Castroviejo, 1992; Rubido-Bará et al., 2010), thus raising the question of the role of genome duplications in driving different distributional patterns and successful establishment and spread of neopolyploids. However, since no neotetraploids have been found in natural diploid populations of $J$. maritima (Castro et al., 2015; Castro, 2018) to study the effects of genome duplications per se, it is fundamental to synthesize tetraploids in the laboratory. For this, an optimal procedure to obtain synthetic tetraploids in this species needs to be developed.

Considering all the above, the main objective of this study was to develop a protocol to obtain synthetic tetraploids from wild diploid plants of J. maritima. Additionally, a methodology which synchronizes seed germination is also presented. Specifically, we wanted to address the following questions: (1) what is the effect of different colchicine concentrations in survival and polyploidization success of J. maritima seedlings? (2) What is the effect of different seedling ages on seedling survival and successful induction of neotetraploids? (3) Do the differences in polyploidization success vary be- 
tween populations? As a result of this study, we propose a protocol to induce synthetic polyploids, with prospects of being applied to other polyploid complexes without neotetraploids detected in nature.

\section{Materials and methods}

\subsection{Study species and field sampling}

Fruiting heads of J. maritima were collected in July 2013 in four natural populations previously confirmed to be homogenously diploid (Castro et al., 2015; Castro, 2018), namely Population 1 - Lariño (POP1; 42.77103, -9.12227), Population 2 - Fisterra (POP2; 42.90851, -9.27328), Population 3 - Neriña (POP3; 43.00983, -9.26141) and Population 4 - Soesto (POP4; 43.2124, -9.02343), all in La Coruña, Spain. Within each population, fruiting heads from 40 mother plants, at least $4 \mathrm{~m}$ apart, were collected in individual paper bags. Seeds were air dried, cleaned from fruiting heads and harvested in labelled microtubes. Several seeds per mother plant (hereafter denoted as seed family) and several mother plants per population were used to study germination rates and explore differences in polyploidization success between populations and seed families. POP1 and POP2 were used in both germination and induction studies, POP3 was only used in the germination studies, and POP4 was only used for induction assays due to low seed availability.

\subsection{Seed germination}

Basic information about germination patterns is fundamental to determine the correct seedling stage for induction. Because no information was available on the germination patterns of J. maritima, a preliminary germination trial focused on obtaining high germination rates and, more importantly, synchronized germination was made in January 2014. For this, 30 seeds from 20 mother plants from three populations were placed to germinate in individual Petri dishes with moist filter paper (POP1, POP2 and POP3) and were subjected to four treatments varying in exposure to cold: (1) conditioned directly in a growth chamber (without cold treatment); (2) conditioned for 3 days at $4{ }^{\circ} \mathrm{C}$ in the dark and then transferred to the growth chamber; (3) conditioned for 1 week at $4{ }^{\circ} \mathrm{C}$ in the dark and then transferred to the growth chamber; (4) conditioned for 2 weeks at $4{ }^{\circ} \mathrm{C}$ in the dark and then transferred to the growth chamber. The conditions of the growth chamber were $16: 8 \mathrm{~h}$ (light/dark) photoperiod with $24^{\circ} \mathrm{C}$ of temperature. Petri dishes were watered when necessary (usually after transference to the growth chamber they were watered every 2 days). Seed germination was monitored for 1 month every day during the first 2 weeks after transference to the growth chamber and every 2 days afterwards. Total germination rates were calculated for each population and treatment as the percentage of seeds that germinated from the total number of seeds placed in the Petri dish. The time needed to reach $50 \%$ of total germination rate (T50) was calculated for each mother plant, enabling to characterize each treatment and population regarding the rate and pace of seed germination: lower T50 values would indicate higher germination synchrony, while higher T50 values would imply a germination extended over longer periods of time. The protocol that resulted in a higher number of seedlings in similar development stages at the moment of polyploid induction was selected for synthetic polyploids induction.

\subsection{Synthetic induction of polyploids}

Synthetic polyploids were induced directly in young seedlings from three natural populations (POP1, POP2 and POP4) by submerging them in aqueous solutions of colchicine (Husband et al., 2008). Two induction trials were made, one testing different colchicine concentrations and another testing different seedling ages. In the first trial, 3day-old seedlings (T1) were used, presenting fully expanded cotyledons and exposing the apical meristem, as well as bearing sufficiently smaller roots that could be manipulated without damage. Up to 30 seedlings per mother plant and 40 mother plants per population were submerged in $0.1 \%$, $0.2 \%, 0.5 \%$ or $1.0 \%$ aqueous colchicine solutions and left in the fume hood overnight for $14 \mathrm{~h}$. An additional set was submerged in sterile $\mathrm{ddH}_{2} \mathrm{O}$ for control. The seedlings were then rinsed 5 times with sterile $\mathrm{ddH}_{2} \mathrm{O}$. In the second trial, we tested the success of polyploid induction when using seedlings with different ages. For this, 2-week-old seedlings (T2) were subjected to a second induction trial using $0.5 \%$ colchicine concentration, following the procedure described above and subsequently compared with the 3-dayold seedlings subjected to the same colchicine concentration. In both cases, after induction, seedlings were carefully transplanted directly to a multi-pot tray containing commercial standard soil. The seedlings were maintained in the greenhouse, watered daily and seedling mortality was monitored weekly.

\subsection{Flow cytometry analyses}

After 6 months of the induction trial, all the plants that survived were analysed with flow cytometry to estimate genome size and DNA ploidy levels. At this stage, plants already had 6-8 leaves enabling the harvest of one fresh leaf for flow cytometric analyses. Fresh leaves were used to prepare the nuclear suspension following the protocol of Galbraith and co-authors (Galbraith et al., 1983), by simultaneously chopping the plant material of the sampled plant with leaf tissue of Solanum lycopersicum "Stupické" (internal reference standard, $2 \mathrm{C}=1.96 \mathrm{pg}$, S.l.; Doležel et al., 1992). Nuclei were isolated in $1 \mathrm{~mL}$ of woody plant buffer (WPB; Loureiro et al., 2007) and filtered through a $50 \mu \mathrm{m}$ nylon filter. Then, $50 \mu \mathrm{g} \mathrm{mL}^{-1}$ propidium iodide and $50 \mu \mathrm{g} \mathrm{mL}^{-1}$ RNAse were added to the sample to stain the DNA and de- 
grade double-stranded RNA, respectively. The sample was analysed in Partec CyFlow Space flow cytometer (532 nm green solid-state laser, $30 \mathrm{~mW}$; Partec GmbH., Görlitz, Germany). Partec FloMax software v2.4d (Partec GmbH, Münster, Germany) was used to obtain the following graphics: fluorescence pulse integral in linear scale (FL); forward light scatter (FS) vs. side light scatter (SS), both in logarithmic (log) scale; FL vs. time; and FL vs. SS in log scale. DNA ploidy levels were inferred for each individual plant based on the chromosome counts and respective genome sizes (cytotype: mean $\pm \mathrm{SD}$; diploids: $2 \mathrm{n}=2 \mathrm{x}=2.98 \pm 0.07$ picograms; tetraploids: $2 \mathrm{n}=4 x=6.06 \pm 0.11 \mathrm{pg}$; Fig. 1a-b). According to this, each plant was classified as DNA diploid, DNA tetraploid, DNA octoploid, DNA aneuploid, diploidtetraploid mixoploids and tetraploid-octoploid mixoploids. A plant was classified as mixoploid when (1) the second largest peak had more than $25 \%$ of the events of the plant sample, and/or (2) when a third peak corresponding to nuclei in phase $\mathrm{G}_{2}$ of mitosis of the subpopulation of cells with the higher ploidy level is detected (Ochatt, 2006).

\subsection{Statistical analyses}

General linear models (GLMs) and generalized linear mixed models (GLMMs) were used to analyse differences in germination rates among treatments and populations. First, we explored overall differences in germination rates and in T50 among treatments, by defining germination treatment as a fixed factor, population as a random factor, and germination rate and T50 as response variables. Germination rates were arccosine transformed. A Gaussian distribution with an identity link function and a Poisson distribution with a $\log$ link function were used to model germination rate and T50, respectively. Differences among populations and colchicine treatments nested within populations were also tested as fixed factors, with germination rate and T50 as response variables, as described above.

GLMMs were also used to analyse differences in survival and induction rates among colchicine treatments, populations and seedling ages. First, we explored overall differences in survival and induction success among colchicine treatments, defining colchicine concentration as fixed factor, population and mother plant as random factors, and survival and induction success as response variables. A binomial distribution with a logit link function was used to model response variables. Second, because population could impact the response of the plants, we also explored differences among populations and colchicine treatments nested within populations as fixed factors, including, as above, the mother plant as a random factor, and survival and induction success as response variables. Finally, a similar approach was used to explore differences in survival and induction success among seedlings with different ages and populations. When significant differences were observed, post hoc tests for multiple comparisons were performed.
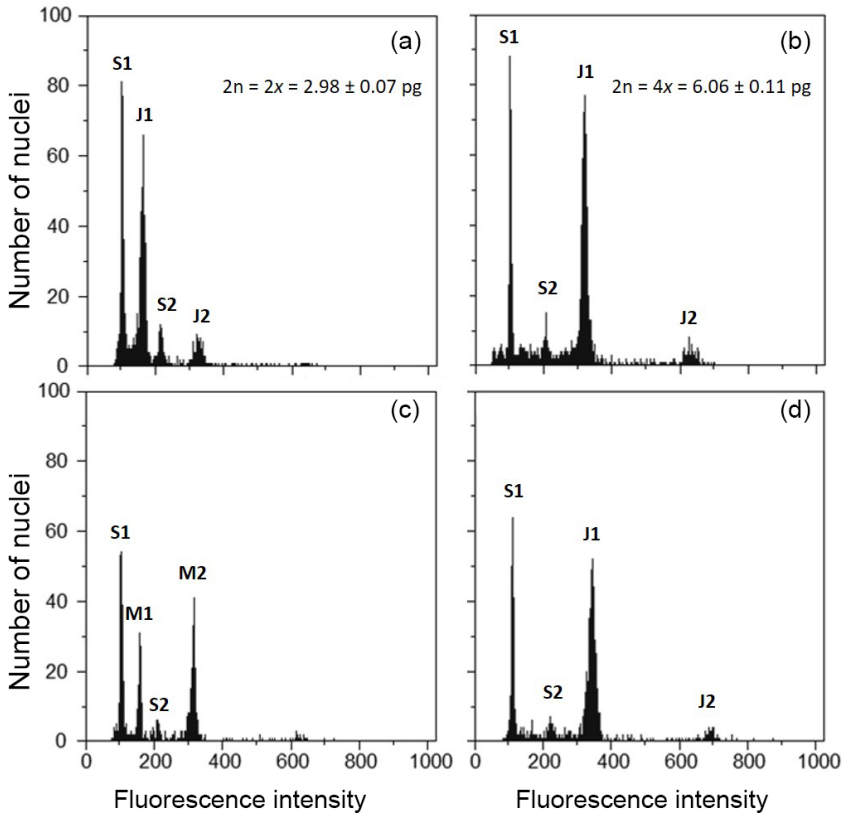

Figure 1. Flow cytometric histograms of J. maritima genome size analyses. Histograms of relative propidium iodide fluorescence intensity of nuclei obtained from fresh leaves of $S$. lycopersicum "Stupické" (as reference standard, 2C $=1.96 \mathrm{pg}$ ) and J. maritima: (a) natural diploid J. maritima; (b) natural tetraploid J. maritima; (c) diploid-tetraploid mixoploid individual obtained after colchicine treatment; and (d) DNA tetraploid individual obtained after colchicine treatment. $S 1$ and $S 2$ correspond to $G_{1}$ and $G_{2}$ peaks of S. lycopersicum, respectively; J1 and J2 correspond to peak $\mathrm{G}_{1}$ and $\mathrm{G}_{2}$ of $J$. maritima, respectively; $\mathrm{M} 1$ and $\mathrm{M} 2$ correspond to nuclei with $2 \mathrm{C}$ and $4 \mathrm{C}$ genome size values, respectively. Mean genome size values $( \pm \mathrm{SD})$ of natural diploid and tetraploid populations of J. maritima are provided in the respective histograms.

The analyses were performed in $\mathrm{R}$ software version 3.0.1 (R Core Development Team, 2016) using the packages "car" for Type III analysis of variance (Fox et al., 2015), "Ime4" for generalized linear models (Bates et al., 2014) a nd "multcomp" for multiple comparisons after Type III analysis of variance (Hothorn et al., 2017).

\section{Results}

\subsection{Seed germination}

J. maritima presented germination rates of $93.1 \pm 0.5 \%$ (mean $\pm \mathrm{SE}$ ), on average. Overall, germination rates differed significantly among treatments $\left(F_{3,236}=4.47, P<\right.$ 0.001 ), with cold treatments increasing total germination rates $(P<0.05)$ (Appendix A). However, when analysing in a nested design the differences became less evident: while significant differences were observed among populations $\left(F_{2,228}=7.93, P<0.001\right.$; population: mean $\pm \mathrm{SE}$, POP1: $89.6 \pm 1.1 \%$, POP2: $96.3 \pm 0.6 \%$, POP3: $93.3 \pm$ 

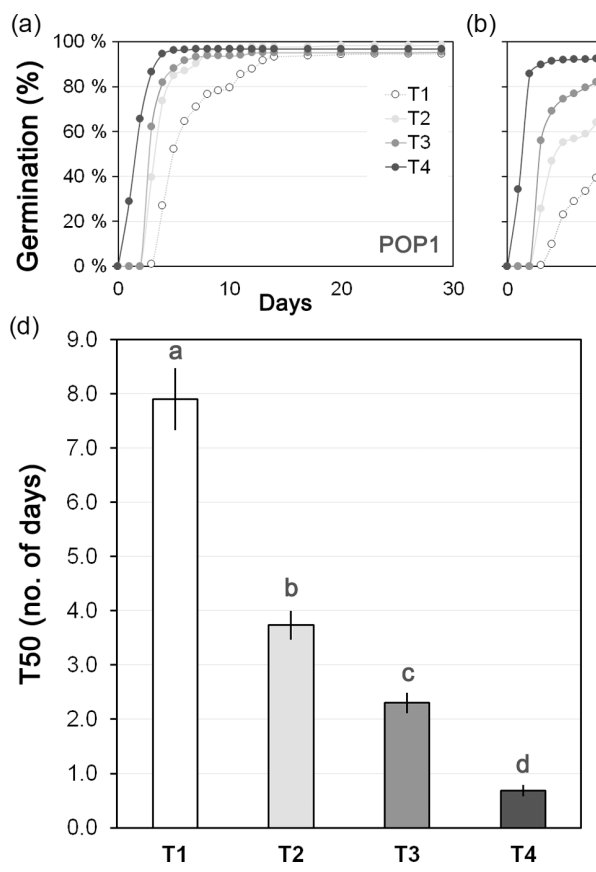

(b)

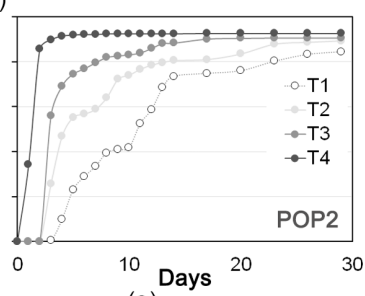

(c)

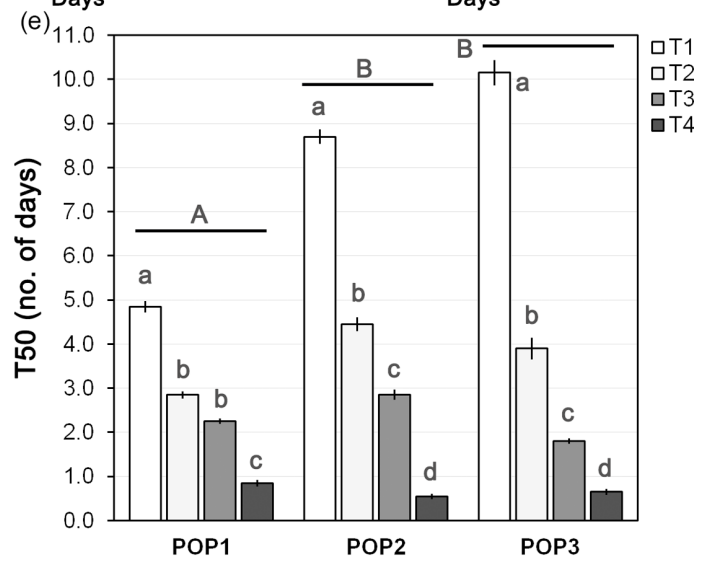

Figure 2. Seed germination of J. maritima diploid populations under different germination conditions. Seed germination (in $\%$ ) over 1 month after different cold treatments is provided for: (a) population 1 (POP1), (b) population 2 (POP2) and (c) population 3 (POP3). The time needed to reach $50 \%$ of total germination rate (T50; mean $\pm \mathrm{SE}$; in number of days) is given for (d) each treatment and (e) each treatment within each studied population. Treatment before transfer to the growth chamber: $\mathrm{T} 1$, without cold treatment; $\mathrm{T} 2,3 \mathrm{days}$ at $4{ }^{\circ} \mathrm{C}$; T3, 1 week at $4{ }^{\circ} \mathrm{C}$; and T4, 2 weeks at $4{ }^{\circ} \mathrm{C}$. Different lower-case letters denote significant differences among treatments, including the total comparison (in d) and among treatments within populations (in e) at $P<0.05$; different upper-case letters denote significant differences among populations at $P<0.05$.

$0.9 \%)$, among treatments within populations the differences were near the significance level $\left(F_{9,228}=1.93, P=0.05\right)$, with the subsequent multiple comparison tests showing no significant differences $(P>0.05)$ (Appendix A).

Although the total germination rates were high overall among treatments, which enabled seedlings to be easily obtained for the induction experiments, the largest differences were observed in the pace of germination, with the increased exposure to cold increasing the germination speed (Fig. 2ac). This pattern was reflected in the T50 values we obtained (Fig. 2d-e). The T50 differed significantly among treatments $\left(\chi_{3}^{2}=362.83, P<0.001, n=240\right)$, from being as slow as $7.9 \pm 0.6$ days without cold treatment to as fast as $0.7 \pm 0.1$ days with the longest cold treatment (Fig. $2 \mathrm{~d}$ ). Differences were also observed among populations $\left(\chi_{2}^{2}=\right.$ 36.59, $P<0.001$ ), with POP2 having significantly lower T50 than the other two populations $(P<0.05$; population: mean \pm SE, POP1: $3.7 \pm 0.02 \%$, POP2: $2.7 \pm 0.02$, POP3: $4.1 \pm 0.06)$ and among treatments within populations $\left(\chi_{9}^{2}=\right.$ 363.40, $P<0.001$ ), with T50 showing the same patterns, i.e. significantly decreasing with the increased exposure to cold $(P<0.05$; Fig. 2e).

\subsection{Synthetic polyploidy induction - colchicine concentrations}

An overall survival rate of $11.5 \pm 0.7 \%$ and a tetraploid conversion rate of $35.6 \pm 2.9 \%$ of the surviving plants was obtained in J. maritima. Survival of control seedlings submerged in $\mathrm{ddH}_{2} \mathrm{O}$ was $100 \%$, thus indicating that mortality was mainly due to the colchicine treatment. Survival rates were variable depending on the colchicine treatment and seed origin (i.e. population), while conversion rates were similar across these factors (i.e. colchicine treatment and population), being surprisingly high.

Seedling survival varied between $4.5 \pm 0.9 \%$ and $19.5 \pm$ $1.5 \%$ (mean $\pm \mathrm{SE}$; for treatments with $1.0 \%$ and $0.1 \%$ of colchicine, respectively) and differed significantly among colchicine treatments $\left(\chi_{3}^{2}=35.69, P<0.001, n=\right.$ $2341)$, with survival significantly decreasing with increased colchicine concentration $(P<0.05)$ (Fig. 3a). Differences were also observed among populations $\left(\chi_{2}^{2}=12.40, P=\right.$ 0.002), with one of the populations (POP2: $18.6 \pm 1.4 \%$ ) having significantly higher survival rates than the remaining populations (POP1: $7.3 \pm 0.9 \%$ and POP4: $8.3 \pm 1.0 \% ; P<$ $0.05)$, and among concentrations within population $\left(\chi_{9}^{2}=\right.$ 39.89, $P<0.001)$. Again, survival decreased with increased 

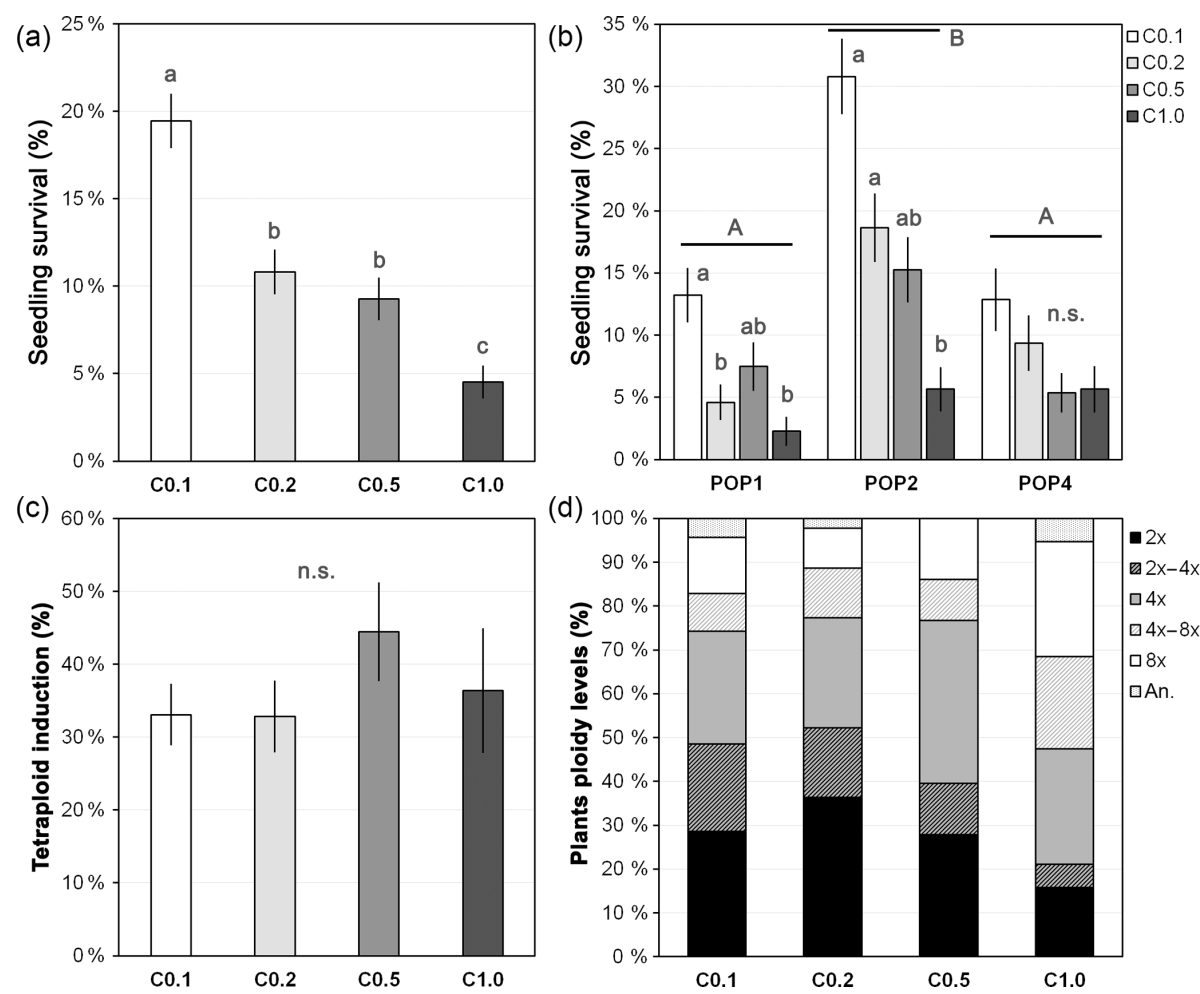

Figure 3. Colchicine treatment effect on seedling survival, synthetic tetraploid induction and ploidy levels of treated seedlings. (a) Overall seedling survival for each treatment with different colchicine concentrations, (b) seedling survival for each treatment within each studied population, (c) synthetic tetraploid induction rate per treatment and (d) ploidy levels of the plants after treating seedlings with different colchicine concentrations. Treatment with different colchicine concentrations: $\mathrm{C} 0.1$, colchicine at $0.1 \%$; $\mathrm{C} 0.2$, colchicine at $0.2 \%$; $\mathrm{C} 0.5$, colchicine at $0.5 \%$; C1.0, colchicine at $1.0 \%$. Ploidy levels: $2 x$, DNA diploid; $2 x-4 x$, diploid-tetraploid mixoploids; $4 x$, DNA tetraploid; $4 x-8 x$, tetraploid-octoploid mixoploids; $8 x$, DNA octoploid; an., DNA aneuploids. Values are given in percentage as mean and standard error of the mean (in $\mathbf{a}-\mathbf{c}$ ) or percentage from the total (in $\mathbf{d}$ ). Different lower-case letters denote significant differences among treatments, including the total comparison (in a) and among treatments within population (in $\mathbf{b}$ ) at $P<0.05$; different upper-case letters denote significant differences among populations at $P<0.05$; n.s. denotes non-significant differences between treatments at $P>0.05$.

colchicine concentration, although this effect was only significant in POP1 and POP2 $(P<0.05$; Fig. 3b).

The induction success, measured through the production of tetraploids, did not differ significantly between colchicine concentrations $\left(\chi_{3}^{2}=2.57, P=0.463, n=267\right)$, although there was a trend of increasing tetraploid induction (Fig. 1c) with increased colchicine concentration (Fig. 3c-d). The lack of differences was also observed among populations $\left(\chi_{2}^{2}=\right.$ $0.59, P=0.743)$ and colchicine concentrations within population $\left(\chi_{9}^{2}=8.35, P=0.499\right)$. Still, although the proportion of synthetic tetraploids did not differ significantly between colchicine treatments, the ploidy levels detected in the seedlings were variable among colchicine treatments. The higher concentration tested originated a lower percentage of DNA diploid and diploid-tetraploid mixoploid individuals (Fig. 1d) and a higher percentage of individuals with higher ploidies, including DNA octoploids and tetraploid-octoploid mixoploid plants (Fig. 3d).

\subsection{Synthetic induction of polyploids - seedling age}

The age at which the seedling was manipulated significantly affected the survival rates $\left(\chi_{1}^{2}=23.60, P<0.001\right.$, $n=1817$ ), with younger seedlings having significantly lower survival rates than the older ones (mean \pm SE, $9.3 \pm 1.2 \%$ and $19.8 \pm 1.1 \%$, respectively; $P<0.05$; Fig. 4a). However, a high variability was also observed due to population, with significant differences being observed among populations $\left(\chi_{2}^{2}=17.43, P=0.001\right)$, with one of the populations (POP4: $10.5 \pm 3.6 \%$ ) having lower survival rates than the other two (POP1: $16.3 \pm 6.2 \%$ and POP2: $16.9 \pm 1.1 \% ; P<$ $0.05)$. Also, significant differences were observed between seedling ages within population $\left(\chi_{3}^{2}=35.24, P<0.001\right)$ (Fig. 4b), with the overall pattern of increasing survival with increased age being evident in each population. When populations were analysed separately, the differences were only significant for POP1 and POP4 ( $P<0.05$; Fig. 4b).

Once again, no differences were observed in the percentage of synthetic tetraploids obtained between the two groups 

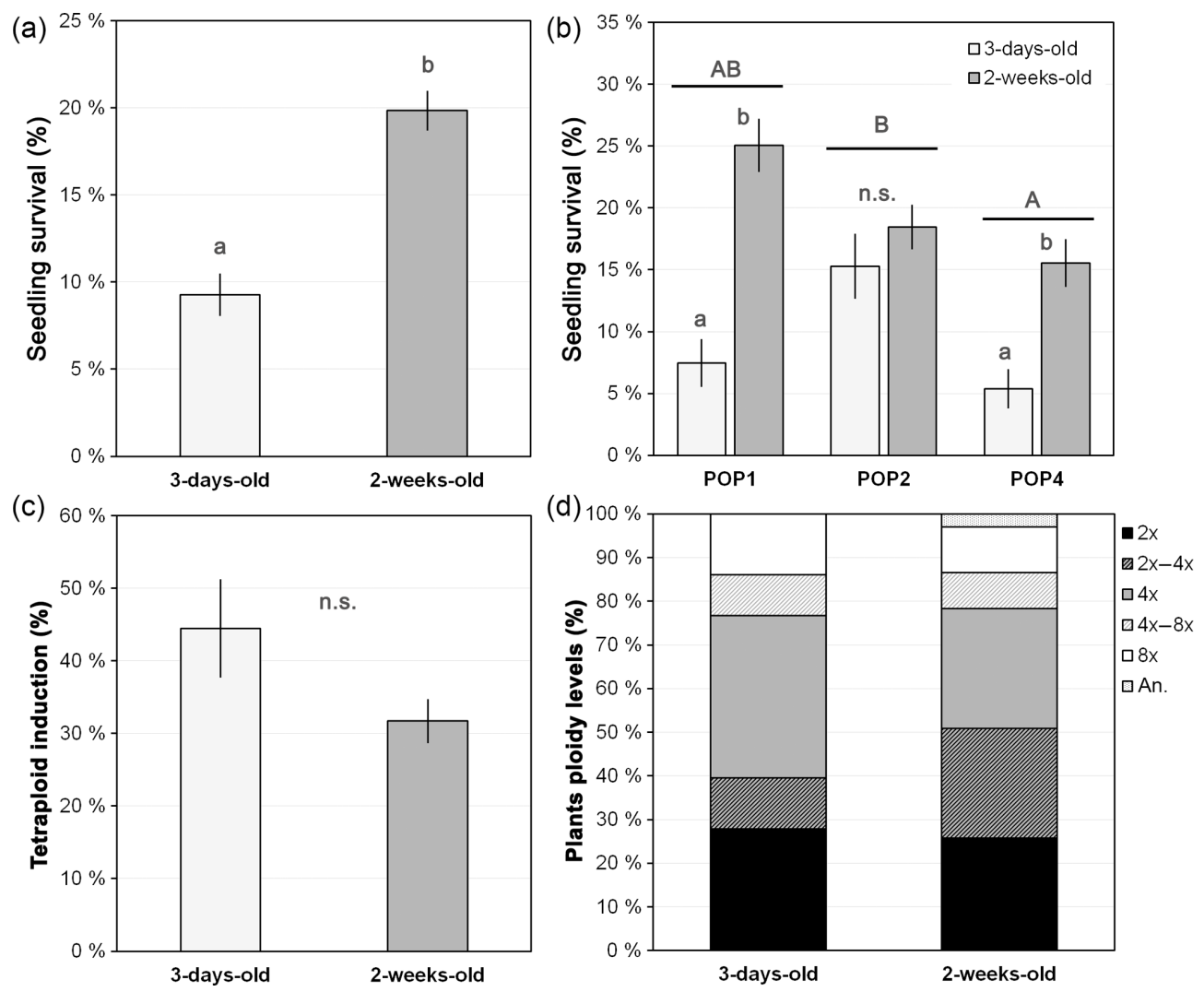

Figure 4. Seedling age effect on seedling survival, synthetic tetraploid induction and ploidy levels of treated seedings. (a) Overall seedling survival for each age category; (b) seedling survival for each age category within each studied population; (c) percentage of synthetic tetraploid induction per age category; and (d) ploidy level of the plants after treating seedlings with different ages with colchicine. Age categories denote the age at which the seedling was treated with colchicine: 3-day-old and 2-week-old seedlings. Ploidy levels: $2 x$, DNA diploid; $2 x-4 x$, diploid-tetraploid mixoploids; $4 x$, DNA tetraploid; $4 x-8 x$, tetraploid-octoploid mixoploids; $8 x$, DNA octoploid; an., DNA aneuploids. Values are given in percentage as mean and standard error of the mean (in a-c) or percentage from the total (in d). Different lowercase letters denote significant differences among treatments, including the total comparison (in a) and among treatments within population (in b) at $P<0.05$; different upper-case letters denote significant differences among populations at $P<0.05$; n.s. denote non-significant differences between treatments at $P>0.05$.

varying in seedling age $\left(\chi_{1}^{2}=2.65, P=0.104, n=130\right)$, although there was a pattern of a lower percentage of tetraploid induction with increased age (Fig. 4c). The lack of differences was also consistent among populations $\left(\chi_{2}^{2}=0.29\right.$, $P=0.865)$ and among colchicine concentrations within population $\left(\chi_{3}^{2}=4.83, P=0.184\right)$. Although no statistical differences were observed, older seedlings subjected to colchicine treatment seemed to produce a higher percentage of diploid-tetraploid mixoploids plants (marginally significant: $\left.\chi_{1}^{2}=3.61, P=0.057\right)$ instead of tetraploid individuals (Fig. 4d).

\section{Discussion}

J. maritima is a diploid-tetraploid complex ideal for exploring the role of genome duplications in the success of polyploid lineages, but neotetraploids have never been found in nature (Castro et al., 2015; Castro, 2018). In this study we tested the effect of different colchicine concentrations and seedling ages in polyploid induction and the successful production of synthetic tetraploids from several populations and seed families of the diploid J. maritima.

The success of the polyploidization process depends on numerous factors, such as the type of explant, the anti-mitotic agent used and its ability to penetrate the cells, the concentration applied and the duration of the exposure (Ascough et al., 2008, and references therein). The protocols used to induce polyploidy can start from a wide variety of plant materials, in which the presence of active meristems is fundamental to achieve successful polyploidization. However, in the case of ecological studies the most frequently used explants are seeds (Pringle and Murray, 1992; Walters and Wehne, 2002; Thompson et al., 2010) or seedlings (Husband et al., 2008; Tate et al., 2009; Pavlíková et al., 2017), as such plant materials enable us to introduce numerous genotypes in the study and, thus, account for the genetic variability of the diploid (or 
lower ploidy level) parental populations. The use of seeds and seedlings as explants are also particularly suitable for species with small seeds such as Jasione. However, the use of seedlings requires the development of a protocol that enables the obtainment of a high number of explants ready for polyploidization induction, especially if polyploidization rates are low, and ideally with synchronized development. In this study, a protocol of synchronization of seed germination using a cold treatment is proposed, in which most of the seeds germinated within a few days after being transferred from cold conditions to optimal growing conditions. Although we observed that the seeds of J. maritima germinated at room temperature, the cold treatment seemed to synchronize seed germination by holding the development of the seedling until the temperature conditions are favourable (Garcia et al., 2006; Ribeiro and Costa, 2015). This enabled us to obtain a high number of seedlings in a similar developmental stage for induction of polyploidization. It is worth noticing that high levels of germination were observed in this species, averaging $93 \%$, although values varied significantly between $84.9 \%$ and $98.5 \%$ according to the population and treatment.

Colchicine has been successfully used with a wide range of concentrations depending on the studied species (e.g. from very low concentrations, with $0.00001 \%$ in Lychnis senno (Chen et al. 2006) to very high concentrations, with $1.5 \%$ in Chaenomeles japonica, Stanys et al., 2006). The literature suggests that while the treatments based on higher concentrations of colchicine and/or exposure for longer periods are necessary for successful polyploidization, it was also verified that these treatments are very aggressive, being in many cases lethal to the explants (e.g. Acacia crassicarpa, Lam et al., 2014; C. japonica, Stanys et al., 2006; Cucumis spp., Walters and Wehner, 2002; L. senno, Chen et al., 2006; Watsonia lepida, Ascough et al., 2008). Consequently, the ideal protocol must consider a balance between survival and successful polyploidization. Here, after testing a series of increasing concentrations (namely, $0.1 \%, 0.2 \%$, $0.5 \%$ or $1.0 \%$ aqueous colchicine solutions), we observed that the most detrimental step to produce synthetic tetraploids in J. maritima was the lethality of the seedlings after the colchicine treatment. Survival of J. maritima seedlings was always lower than $19.5 \%$, decreasing significantly with increased colchicine concentrations to survival rates of $4.5 \%$. This result was not surprising given the fragile nature of the seedlings. Similar patterns have been reported in the available studies (Chen et al., 2006; Stanys et al., 2006; Ascough et al., 2008; Ntuli and Zobolo, 2008; Pavlíková et al., 2017). Also, expectedly, survival rates were also affected by seedling age with older seedlings presenting higher survival rates (increasing survival from $9.3 \%$ to $19.8 \%$ when subjected to the same colchicine concentration).

Despite the low survival rates after exposure to the antimitotic agent, we obtained high polyploidization rates in comparison with other studies (e.g. $11 \%$ in Ascough et al., 2008; $13 \%$ in Lam et al., 2014; $13 \%$ in Sakhanokho et al., 2009). Surprisingly, the conversion rates did not differ among colchicine treatments $(35.6 \%$ of neotetraploids from the total surviving seedlings) or between seedling ages $(34.0 \%)$. This result was surprising because most studies up to date showed increased polyploidization success with increased concentrations or exposure to colchicine (e.g. Chen et al., 2006; Stanys et al., 2006; Ascough et al., 2008; Ntuli and Zobolo, 2008; Sakhanokho et al., 2009; Pavlíková et al., 2017). Additionally, some patterns have emerged when analysing the ploidy level of the surviving plants. First, the seedlings subjected to higher colchicine concentrations tended to have lower percentages of diploid and diploidtetraploid mixoploids; instead, they presented higher percentages of tetraploid-octoploid mixoploids and even higher amounts of octoploid individuals than the set of seedlings subjected to the lower colchicine concentrations, with $0.5 \%$ colchicine apparently being the turnover point. Second, when comparing seedlings of different ages subjected to $0.5 \%$ of colchicine, older seedlings tended to result in lower percentages of tetraploid plants and higher percentages of diploidtetraploid mixoploids, suggesting that older seedlings did not convert as efficiently as younger ones. Thus, selecting the ideal developmental stage and age at which to subject the seedling to the anti-mitotic agent seems important; despite this, these factors have seldom been tested. Instead, most studies using seedlings exposed the explants when the cotyledons were fully expanded (Omran and Mohammad, 2008; Ye et al., 2010; Zhang et al., 2010) so that the meristems in active division are fully exposed to the colchicine solution. Because of the very limited information on explant age available in the low number of protocols that have used seedlings, it is difficult to compare polyploidization success rates across studies.

As referred to above, in some of the concentrations, besides the formation of synthetic polyploids, many of the treated seedlings resulted in plants presenting tissues with mixed ploidy patterns, especially diploid and tetraploid in the lower colchicine concentrations, or tetraploid and octoploid in the highest concentration. The creation of chimeric individuals is quite common in polyploidy induction studies (Pringle and Murray, 1992; Väinölä and Repo, 2001; Ascough et al., 2008) but at variable rates, and it has been attributed to the use of a multicellular tissue and an uneven penetration of the colchicine to the seedling tissue. Considering the high number of plants involved in the study, it was not possible to follow the progress of those mixoploid plants.

So far, most of the studies that compared natural diploids and polyploids with synthetic polyploids were based on seeds or seedlings that originated from a single population or did not account for this factor (Husband et al., 2008; Maherali et al., 2009; Griffin et al., 2012; Husband et al., 2016). Despite that, differences between synthetic polyploids of a different origin can be expected, as revealed by Oswald and Nuismer (2011) in $H$. grossulariifolia. In our study, most of the population-related differences were observed in seed germi- 
nation and seedling survival. A recent study by Münzbergová (2017), also shown for interactions between population and some of the traits that were compared in natural and synthetic tetraploids of Vicia cracca (e.g. plant size at 2 weeks, the measures of seed production and stomata size). This suggests that the colchicine effects are context dependent and probably result from the varying genetic composition of each population. Thus, the use of multiple populations should be regarded as an important aspect when developing a new protocol to induce polyploidy, at least in ecology-related studies.

Although it is an invaluable tool, in particular when no neopolyploids are found in nature, the production of synthetic polyploids presents some drawbacks that should be highlighted. In some studies, diploid progenitors and synthetic polyploids expressed different phenotypes, including production of a higher amount of biomass, larger organs and higher amounts of secondary metabolites, among other effects (Hassan and Wazuddin, 2000; Jaskani et al., 2005; Contreras et al., 2007; Cohen et al., 2013). However, these changes were shown to result not only from polyploidization, but from the combination of polyploidization and of antimitotic compound effects (Ramsey and Schemske 2002). To circumvent this problem, Husband et al. (2008) suggested that the phenotypic effects of the anti-mitotic agents could be eliminated by using the second-generation of synthetic polyploids (either by selfing or by intercrossing two synthetic polyploids of the same population) and by analysing the performance of the offspring of these parents. Still, it is not clear if the genetic effects of anti-mitotic substances persist. A recent study by Münzbergová (2017) suggested that plant performance can also be affected by colchicine treatment, even in the second generation.

\section{Conclusions}

The survival and polyploidization rates obtained here suggest that the lowest colchicine concentration $(0.1 \%)$ applied to younger seedlings ( 3 days old) was the most successful treatment, as it enabled a higher survival of the seedlings than the more aggressive concentrations, while maintaining a relatively high induction rate, leading to an overall higher number of synthetic tetraploids. The use of older seedlings might increase survival rates, but it seems to compromise a complete tetraploid conversion by generating many diploidtetraploid mixoploid plants.

Data availability. Data are publicly accessible at Zenodo with the following https://doi.org/10.5281/zenodo.1340550. 
Appendix A: Seed germination of J. maritima diploid populations under different germination conditions
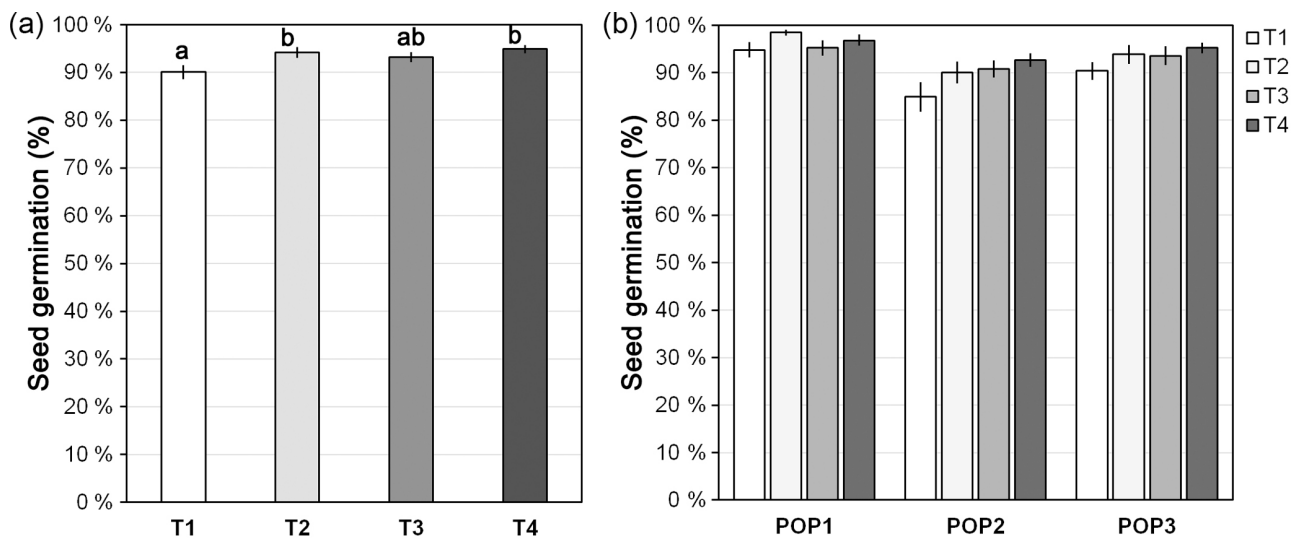

Figure A1. Seed germination (mean $\pm \mathrm{SE}$; in \%) for (a) different cold treatments and (b) each treatment within each studied population. Treatment before transfer to the growth chamber: T1, without cold treatment; T2, 3 days at $4{ }^{\circ} \mathrm{C} ; \mathrm{T} 3,1$ week at $4{ }^{\circ} \mathrm{C}$; and $\mathrm{T} 4,2$ weeks at $4^{\circ} \mathrm{C}$. Different lower-case letters denote significant differences among treatments at $P<0.05$. 
Author contributions. SC and JL conceived and designed the experiments. All authors performed the experiments, wrote the paper and approved the final manuscript.

Competing interests. The authors declare that they have no conflict of interest.

Acknowledgements. The authors are thankful to Daniela Tavares, Lucie Mota and Patricia Caldeirinha for their help in seedling transplants. This research was supported by project ReNATURE (Centro 2020, Centro-01-0145-FEDER-000007) and by $\mathrm{POPH} / \mathrm{FSE}$ funds by the Portuguese Foundation for Science and Technology (FCT) with a doctoral grant to MC (SFRH/BD/89910/2012), and an exploratory project and starting grant to SC (IF/01267/2013).

Edited by: Jutta Stadler

Reviewed by: two anonymous referees

\section{References}

Allum, J. F., Bringloe, D. H., and Roberts, A. V.: Chromosome doubling in a Rosa rugosa Thunb. hybrid by exposure of in vitro nodes to oryzalin: the effects of node length, oryzalin concentration and exposure time, Plant Cell Rep., 26, 1977-1984, 2007.

Ascough, G. D., Van Staden, J., and Erwin, J. E.: Effectiveness of colchicine and oryzalin at inducing polyploidy in Watsonia lepida NE Brown, HortScience, 43, 2248-2251, 2008.

Barker, M. S., Arrigo, N., Baniaga, A. E., Li, Z., and Levin, D. A.: On the relative abundance of autopolyploids and allopolyploids, New Phytol., 210, 391-398, 2015.

Bates, D., Maechler, M., Bolker, B., and Walker, S.: lme4: linear mixed-effects models using Eigen and S4, available at: http:// CRAN.R-project.org/package=lme4 (last access: January 2018), 2014

Bretagnolle, F. and Lumaret, R.: Bilateral polyploidization in Dactylis glomerata L. subsp. lusitanica: occurrence, morphological and genetic characteristics of first polyploids, Euphytica, 84, 197-207, 1995.

Caperta, A. D., Delgado, M., Ressurreição, F., Meister, A., Jones, R. N., Viegas, W., and Houben, A.: Colchicine-induced polyploidization depends on tubulin polymerization in c-metaphase cells, Protoplasma, 227, 147-153, 2006.

Castro, M.: Evolutionary ecology of polyploids: understanding species coexistence at the contact zones, $\mathrm{PhD}$ thesis, Department of Life Sciences, University of Coimbra, Coimbra, 2018

Castro, S., Loureiro, J., Castro, M., Serrano, M., and Husband, B.: Understanding the ecological consequences of genome duplication, Book of abtracts of EMBO workshop: Mechanisms of plant speciation, 53-54, 2015.

Chen, L. P., Wang, Y. J., and Zhao, M.: In vitro induction and characterization of tetraploid Lychnis senno Siebold et Zucc, HortScience, 41, 759-761, 2006.

Cohen, H., Fait, A., and Tel-Zur, N.: Morphological, cytological and metabolic consequences of autopolyploidization in
Hylocereus (Cactaceae) species, BMC Plant Biol., 13, 173, https://doi.org/10.1186/1471-2229-13-173, 2013.

Comai, L.: The advantages and disadvantages of being polyploid, Nat. Rev. Genet., 6, 836-846, 2005.

Contreras, R. N., Ranney, T. G., and Tallury, S. P.: Reproductive behavior of diploid and allotetraploid Rhododendron L. "Fragrant Affinity", HortScience, 42, 31-34, 2007.

De Kovel, C. G. F. and De Jong, G.: Selection on apomictic lineages of Taraxacum at establishment in a mixed sexual-apomictic population, J. Evol. Biol., 13, 561-568, 2000.

Doležel, J., Sgorbati, S., and Lucretti, S.: Comparison of three DNA fluorochromes for flow cytometric estimation of nuclear DNA content in plants, Physiol. Plant., 85, 625-631, 1992.

Fox, J., Weisberg, S., Adler, D., Bates, D., Baud-Bovy, G., and Ellison, S.: car: Companion to applied regression, available at: http: //CRAN.R-project.org/package=car (last access: January 2018), 2015.

Galbraith, D. W., Harkins, K. R., Maddox, J. M., Ayres, N. M., Sharma, D. P., and Firoozabady, E.: Rapid flow cytometric analysis of the cell cycle in intact plant tissues, Science, 220, 10491051, 1983.

Garcia, J., Barker, D. G., and Journet, E. P.: Seed storage and germination, in: $M$. truncatula protocol chapters, Medicago truncatula Handbook, The Samuel Roberts Noble Foundation, Ardmore, OK, chap. 2, 9 pp., available at: https://www.noble.org/globalassets/docs/medicago-handbook/ seed-storage-germination.pdf (last access: March 2018), 2006.

Gmitter, F. G., Ling, X., Cai, C., and Grosser, J. W.: Colchicineinduced polyploidy in Citrus embryogenic cultures, somatic embryos, and regenerated plantlets, Plant Sci., 74, 135-141, 1991.

Griffin, A. R., Vuong, T. D., Vaillancourt, R. E., Harbard, J. L., Harwood, C. E., Nghiem, C. Q. and Thinh, H. H.: The breeding systems of diploid and neoautotetraploid clones of Acacia mangium Willd. in a synthetic sympatric population in Vietnam, Sex. Plant Reprod., 25, 257-265, 2012.

Hassan, L. and Wazuddin, M.: Colchicine-induced variation of cell size and chloroplast number in leaf mesophyll of rice, Plant Breed., 119, 531-533, 2000.

Heinz, D. J. and Mee, G. W.: Colchicine-induced polyploids from cell suspension cultures of sugarcane, Crop Sci., 10, 696-699, 1970.

Hothorn, T., Bretz, F., Westfall, P., and Heiberger, R. M.: multcomp: Simultaneous Inference in General Parametric Models, available at: http://CRAN.R-project.org/package=multcomp (last access: January 2018), 2017.

Husband, B. C. and Sabara, H. A.: Reproductive isolation between autotetraploids and their diploid progenitors in fireweed, Chamerion angustifolium (Onagraceae), New Phytol., 161, 703-713, 2004.

Husband, B. C., Ozimec, B., Martin, S. L., and Pollock, L.: Mating consequences of polyploid evolution in flowering plants: current trends and insights from synthetic polyploids, Int. J. Plant Sci., 169, 195-206, 2008.

Husband, B. C., Baldwin, S. J., and Suda, J.: The incidence of polyploidy in natural plant populations: major patterns and evolutionary processes, in: Plant genome diversity, edited by: Greilhuber, J., Doležel, J., and Wendel, J. F., Springer-Verlag, Vienna, 2, 255-276, 2013. 
Husband, B. C., Baldwin, S. J., and Sabara, H. A.: Direct vs. indirect effects of whole-genome duplication on prezygotic isolation in Chamerion angustifolium: implications for rapid speciation, Am. J. Bot., 103, 1259-1271, 2016.

Jaskani, M. J., Kwon, S. W., and Kim, D. H.: Comparative study on vegetative, reproductive and qualitative traits of seven diploid and tetraploid watermelon lines, Euphytica, 145, 259-268, 2005.

Jersáková, J., Castro, S., Sonk, N., Milchreit, K., Schödelbauerová, I., Tolasch, T., and Dötterl, S.: Absence of pollinator-mediated premating barriers in mixed-ploidy populations of Gymnadenia conopsea sl (Orchidaceae), Evol. Ecol., 24, 1199-1218, 2010.

Kim, S., Rayburn, A. L., Boe, A., and Lee, D. K.: Neopolyploidy in Spartina pectinata Link: 1. Morphological analysis of tetraploid and hexaploid plants in a mixed natural population, Plant Syst. Evol., 298, 1073-1083, 2012.

Kron, P., Suda, J., and Husband, B. C.: Applications of flow cytometry to evolutionary and population biology, Annu. Rev. Ecol. Evol. S., 38, 847-876, 2007.

Lago, M. E. and Castroviejo, S.: Estudio citotaxonómico de la flora de las costas gallegas, vol. 3 of Cadernos da Área de Ciencias Biolóxicas, Compostela Seminario de Estudos Galegos Santiago Area de Ciencias Biolóxicas, 215 pp., 1992.

Lam, H. K., Harbard, J. L., and Koutoulis, A.: Tetraploid induction of Acacia crassicarpa using colchicine and oryzalin, J. Trop. For. Sci., 347-354, 2014.

Levin, D. A. (Ed.): The role of chromosomal change in plant evolution, Oxford University Press, Oxford, UK, 2002.

Lignowski, E. M. and Scott, E. G.: Effect of trifluralin on mitosis, Weed Sci., 20, 267-270, 1972.

Loureiro, J., Rodriguez, E., Doležel, J., and Santos, C.: Two new nuclear isolation buffers for plant DNA flow cytometry: a test with 37 species, Ann. Bot., 100, 875-888, 2007.

Lumaret, R.: Adaptive strategies and ploidy levels, Acta Oecol., 9, 83-93, 1988.

Maherali, H., Walden, A. E., and Husband, B. C.: Genome duplication and the evolution of physiological responses to water stress, New Phytol., 184, 721-731, 2009.

Manzaneda, A. J., Rey, P. J., Bastida, J. M., Weiss-Lehman, C., Raskin, E., and Mitchell-Olds, T.: Environmental aridity is associated with cytotype segregation and polyploidy occurrence in Brachypodium distachyon (Poaceae), New Phytol., 193, 797805, 2012.

Martin, S. L. and Husband, B. C.: Whole genome duplication affects evolvability of flowering time in an autotetraploid plant, PLoS One, 7, e44784, https://doi.org/10.1371/journal.pone.0044784, 2012.

Martin, S. L. and Husband, B. C.: Adaptation of diploid and tetraploid Chamerion angustifolium to elevation but not local environment, Evolution, 67, 1780-1791, 2013.

Münzbergová, Z.: Colchicine application significantly affects plant performance in the second generation of synthetic polyploids and its effects vary between populations, Ann. Bot., 120, 329-339, 2017.

Ntuli, N. R. and Zobolo, A. M.: Effect of water stress on growth of colchicine induced polyploid Coccinia palmata and Lagenaria sphaerica plants, Afr. J. Biotechnol., 7, 3548-3652, 2008.
Ochatt, S. J.: Flow cytometry (ploidy determination, cell cycle analysis, DNA content per nucleus), in: Cytology and microscopy, edited by: Mathesius, U., Journet, E. P., and Sumner, L. W., Medicago truncatula handbook, Chapter 15, 13 pp., available at: https://www.noble.org/globalassets/docs/ medicago-handbook/flow-cytometry.pdf (last access: March 2018), 2006.

Omran, A. and Mohammad, B.: Polyploidization effect in two diploid cotton (Gossypium herbaceum L. and G. arboreum L.) species by colchicine treatments, Afr. J. Biotechnol., 7, 102-108, 2008.

Oswald, B. P. and Nuismer, S. L.: Neopolyploidy and diversification in Heuchera grossulariifolia, Evolution, 65, 1667-1679, 2011.

Pavlíková, Z., Paštová, L., and Münzbergová, Z.: Synthetic polyploids in Vicia cracca: methodology, effects on plant performance and aneuploidy, Plant Syst. Evol., 303, 827-839, 2017.

Petit, C. and Thompson, J. D.: Species diversity and ecological range in relation to ploidy level in the flora of the Pyrenees, Evol. Ecol., 13, 45-66, 1999.

Pringle, G. J. and Murray, B.: Polyploidy and Aneuploidy in the Tamarillo, Cyphomandra betacea (Cav.) Sendt. (Solanaceae), Plant Breeding, 108, 139-148, 1992.

R Core Development Team: A language and environment for statistical computing. Vienna: R Foundation for Statistical Computing, available at: http://www.R-project.org/ (last access: January 2018), 2016.

Ramsey, J.: Polyploidy and ecological adaptation in wild yarrow, P. Natl. Acad. Sci. USA, 108, 7096-7101, 2011.

Ramsey, J. and Schemske, D. W.: Neopolyploidy in flowering plants, Annu. Rev. Ecol. Syst., 33, 589-639, 2002.

Ribeiro, J. N. S. and Costa, C. S. B.: The effect of temperature regulation on seed germination of the tropical tree Myrsine parvifolia A. DC near its southern limit, S. Afr. J. Bot., 98, 128-133, 2015.

Rubido-Bará, M., Horzales, M., and Villaverde, C.: Dos nuevas subespecies del género Jasione L. (Campanulaceae) en el Noroeste de la Península Ibérica, NACC, 19, 21-31, 2010.

Sakhanokho, H. F., Rajasekaran, K., Kelley, R. Y., and IslamFaridi, N.: Induced polyploidy in diploid ornamental ginger (Hedychium muluense RM Smith) using colchicine and oryzalin, HortScience, 44, 1809-1814, 2009.

Sales, F. and Hedge, I. C.: Jasione L., in: Flora iberica, edited by: Castroviejo, S., Paiva, J., Sales, F., Hedge, I. C., Aedo, C., Aldasoro, J. J., Herrero, A., and Velayos, M., Real Jardín Botánico, C.S.I.C., Madrid, 6, 153-170, 2001.

Segraves, K. A. and Thompson, J. N.: Plant polyploidy and pollination: floral traits and insect visits to diploid and tetraploid Heuchera grossulariifolia, Evolution, 53, 1114-1127, 1999.

Semeniuk, P. and Arisumi, T.: Colchicine-induced tetraploid and cytochimeral roses, Bot. Gaz., 129, 190-193, 1968.

Soltis, D. E., Albert, V. A., Leebens-Mack, J., Bell, C. D., Paterson, A. H., Zheng, C. F., Sankoff, D., de Pamphilis, C. W., Wall, P. K., and Soltis, P. S.: Polyploidy and Angiosperm diversification, Am. J. Bot., 96, 336-348, 2009.

Soltis, P. S.: Ancient and recent polyploidy in angiosperms, New Phytol., 166, 5-8, 2005.

Stanys, V., Weckman, A., Staniene, G., and Duchovskis, P.: In vitro induction of polyploidy in Japanese quince (Chaenomeles japonica), Plant Cell Tiss. Org., 84, 263-268, 2006. 
Tamayo-Ordóñez, M. C., Espinosa-Barrera, L. A., TamayoOrdóñez, Y. J., Ayil-Gutiérrez, B., and Sánchez-Teyer, L. F.: Advances and perspectives in the generation of polyploid plant species, Euphytica, 209, 1-22, 2016.

Tate, J. A., Symonds, V. V., Doust, A. N., Buggs, R. J., Mavrodiev, E., Majure, L. C., Soltis, P. S., and Soltis, D. E.: Synthetic polyploids of Tragopogon miscellus and T. mirus (Asteraceae): 60 years after Ownbey's discovery, Am. J. Bot., 96, 979-988, 2009.

Thompson, D. I., Anderson, N. O., and Van Staden, J.: Colchicineinduced somatic polyploids from in vitro-germinated seeds of South African Watsonia species, HortScience, 45, 1398-1402, 2010.

Väinölä, A. N. U. and Repo, T.: Polyploidization of Rhododendron cultivars in vitro and how it affects cold hardiness, Acta Hortic., 560, 319-322, 2000.

Walters, S. A. and Wehner, T. C.: Incompatibility in diploid and tetraploid crosses of Cucumis sativus and Cucumis metuliferus, Euphytica, 128, 371-374, 2002.
Wood, T. E., Takebayashi, N., Barker, M. S., Mayrose, I., Greenspoon, P. B., and Rieseberg, L. H.: The frequency of polyploid speciation in vascular plants, P. Natl. Acad. Sci. USA, 106, 13875-13879, 2009.

Ye, Y. M., Tong, J., Shi, X. P., Yuan, W., and Li, G. R.: Morphological and cytological studies of diploid and colchicine-induced tetraploid lines of crape myrtle (Lagerstroemia indica L.), Sci. Hortic., 124, 95-101, 2010.

Yu, C. Y., Kim, H. S., Rayburn, A., Widholm, J. M., and Juvik, J. A.: Chromosome doubling of the bioenergy crop, Miscanthus $\times$ giganteus, GCB Bioenergy, 1, 404-412, 2009.

Zhang, W., Hao, H., Ma, L., Zhao, C., and Yu, X.: Tetraploid muskmelon alters morphological characteristics and improves fruit quality, Sci. Hortic., 125, 396-400, 2010.

Zlesak, D. C., Thill, C. A., and Anderson, N. O.: Trifluralinmediated polyploidization of Rosa chinensis minima (Sims) Voss seedlings, Euphytica, 141, 281-290, 2005. 